\title{
MANAGEMENT OF THE SPARE PARTS STORAGE IN THE PRODUCTION PROCESS USING THE MONTE CARLO METHOD
}

doi: 10.2478/cqpi-2019-0035

Date of submission of the article to the Editor: 25/03/2019

Date of acceptance of the article by the Editor: 14/05/2019

\author{
Paweł Ślaski ${ }^{1}$ - orcid id: 0000-0002-1950-9910 \\ ${ }^{1}$ Military University of Technology, Poland
}

\begin{abstract}
The article presents the methodology of controlling stock of spare parts during short-run production of current equipment. A pseudo-random number generator has been developed, whose main task is to estimate the expected value $E$ $(\mathrm{Y})$ of the random variable $\mathrm{Y}$ which is the number of exchanges of a spare part of a particular equipment. By means of a Monte Carlo computer simulation, pseudorandom numbers are generated with a uniform distribution from the interval (0.1). These numbers, based on the cumulative distribution inversion method, are transformed into a form of distribution that uniquely determines the optimal size of spare parts
\end{abstract}

Keywords: spare parts, Monte Carlo simulation, pseudo-random number generator

\section{INTRODUCTION}

The increase of the degree of complexity of modern devices means that ensuring technical readiness requires appropriate construction solutions and a proper exploitation strategy. Four basic strategies can be distinguished:

- with technical support depending on the damage or when it has become unfit,

- with scheduled and preventive maintenance (depending on the time of operation),

- with scheduled and preventive maintenance with diagnosis,

- with support according to the current technical condition (Krzaczak et al., 2012).

Although the actual exploitation strategies combine in a different degree all the modes of action discussed, most benefits are due to exploitation according to their current state. The complexity of problems encountered while using the equipment creates the need to comprehensively address issues related to maintaining them in a technical readiness. For this purpose, one should look for effective diagnostic methods to determine the technical condition of the equipment. When looking for effective diagnostic methods to determine the technical condition of devices, it should be remembered that even the best diagnosis will not fully ensure the functioning of the system, if the issues related to ensuring the supply of spare parts are not taken into account (Krzaczak et al., 2012).

Spare parts are elements, groups of elements or complete products that are used to replace damaged, worn or defective parts, assemblies or products (DIN, 1976). 
According to APICS standards, they are defined as modules, components and elements that are planned to be used without any additional modifications to replace the original parts (APICS, 2004).

Controlling the spare parts inventory should be considered from the point of view of the logistics chain, in which the manufacturer of these parts is the primary link. For him, a number of logistic processes are implemented, from planning needs, production, storage, to parts distribution to the user. The specifics of spare parts stocks requires detailed strategies related to their control. A strategy for maintaining stock of spare parts is a characteristic for each manufacturer. The size of the production series of parts and subassemblies is of great importance here. There is no problem in the case of the need for parts while they are produced on a regular basis for production purposes. The problem arises when parts are produced periodically or when their production is discontinued. The question to consider is whether to keep parts and in what quantities on stock in the warehouse, or to produce if necessary. You should consider and compare the costs of storage and possible production here. In addition to the aforementioned features, the requirements regarding the execution of orders in the spare parts of the recipients should be taken into account: the date of order fulfillment, reliability of delivery, flexibility of deliveries due to the urgency of orders. The timeliness of deliveries of parts and assemblies ensures that unnecessary equipment downtime is avoided. Reliability of deliveries is the most important in such operating systems; where maintenance activities can only be carried out within a strictly defined period of time. Proper implementation of the order can minimize the user's losses resulting from failure or damage to the equipment in the operation process. Orders can be made periodically to supplement stock levels, however, there are often particularly urgent orders. Therefore, there must be flexibility in the execution of orders and ways of transferring spare parts.

The decision on how to control spare parts inventory is related to the system of demand for individual parts, their value and type.

\section{OWN RESEARCH}

The methodology presented in the further part of the article is related to OEM parts (Original Equipment Manufacturer - Original parts are usually produced simultaneously with the production of technological equipment and in the future when there is a need to replace the element) and the decision, according to the following criterion:

What should be the spare part storage during the production of the current device to minimize the expected loss of the equipment user caused by the production line stoppage?

Therefore, the purpose of the article is to develop a methodology for controlling stock of spare parts during short-run current production on a selected example.

For this purpose, the working hypothesis is as follows:

"Determining the optimal size of spare parts during the current production will ensure both the continuity of use of the equipment and its use as intended, as well as minimization of costs for both the manufacturer and the user."

The following research methods were used to develop the methodology:

1. Descriptive statistics.

2. A pseudo-random number generator.

3. Monte Carlo simulation. 
4. Analysis of the costs of spare parts stocks.

Methodology for controlling spare parts inventory

The methodology consists of four stages:

- Determining the probability distribution of a random variable number of spare parts replacements.

- Development of a pseudorandom number generator.

- Determining the optimal size of spare parts.

- Verification of the methodology.

During this stage, it is important to determine the type of distribution of the studied characteristic. With this information, one can estimate the parameters of the distribution. The methods commonly used are: matching the empirical distribution to the theoretical and arraying. In the developed identification model, the distribution type was made by means of arraying. As a result of the measurement of the size of a specific characteristic (number of replacements of spare parts), a typical sequence of quantities is described in the form of an array.

Observations can be divided into (1) (Śląski, 2017):

$$
R=\sqrt{n}
$$

disjoint classes of the same length equal to (2):

$$
L=\frac{x_{n}-x_{1}}{R}
$$

where:

$L, R, n, x_{n}, x_{1}$, denote in turn: class length, number of classes, number of observations, the nth characteristic of the ordered sequence ascending, the first value of the sequence.

In connection with the above, $r$-class $(r=\overline{1, R})$ includes observations that satisfy inequality (3):

$$
x_{1}+(r-1) \cdot L \leq x_{i}<x_{1}+r \cdot L
$$

The mean of class can be determined from formula (4):

$$
x^{s}{ }_{r}=x_{1}+(r-0,5) \cdot L
$$

After determining the number of classes, the number of elements in the class and the means of classes, the class probabilities should be determined using the classic definition. Couples: the mean of the class and the probability of belonging elements to classes, create a probability distribution that can be written in the form of an array.

\section{DETERMINING THE OPTIMAL SIZE OF SPARE PARTS}

Determining the optimal size of spare parts is related to cost analysis. These costs are difficult to determine due to the numerous factors affecting their estimation. Intuitively, it can be deduced that the costs of producing spare parts during the current production will be lower than in the case of their production when the failure occurs. In the first case there may be a risk of unnecessary use of parts, while in the second there is a risk of incurring additional costs related to changing equipment, stopping production lines and loss of user associated with the inability to use the equipment as intended (Sarjusz-Wolski, 2010).

Therefore, the loss incurred by the manufacturer of the device and the user due to the stoppage of the production line created during the production of the spare part is (5): 


$$
W(p, y)= \begin{cases}c_{c} \cdot(p-y), & \text { if } y \leq p \\ \left(c_{s}-c_{c}+c_{d m}\right) \cdot(y-p), & \text { if } y>p\end{cases}
$$

where:

$W(p, y)$ - producer's and user's loss

$c_{c}-$ the purchase price of the part produced during the current production

$c_{S}$ - the purchase price of the part produced in an emergency

$y$ - random variable of parts replacement $(y=0,1,2,3,4,5, \ldots K)$

$p$ - spare parts inventory during the production of the current device

$c_{d m}$ - the cost of machine downtime

For such a defined loss $W(p, y)$, its expected value is presented in accordance with the formula (6):

$$
E(p, y)=\sum_{y=0}^{p} c_{c} \cdot(p-y) \cdot p(y)+\sum_{p+1}^{K}\left(c_{s}-c_{c}+c_{d m}\right) \cdot(y-p) \cdot p(y)
$$

The expected value will be minimized for such a spare part of spare parts that meets condition $(7,8)$ :

$$
c_{S}+c_{d m}>c_{c}
$$

therefore:

$$
\frac{c_{c}}{c_{s}+c_{d m}}<1
$$

so:

or in the general record

$$
0<\frac{c_{c}-c_{s}+c_{d m}}{c_{s}+c_{d m}}<1
$$

where:

$$
F_{p-1}<\frac{c_{c}-c_{s}+c_{d m}}{c_{s}+c_{d m}}<F_{p}
$$

$F_{p-1}, F_{p}$ - distribution of a random variable $y$.

\section{VERIFICATION OF THE METHODOLOGY}

Verification of the methodology was based on statistical data collected during several years of observation of the wear of the spare part in the process of exploitation of specialist military equipment. The probability distribution of the spare parts is presented in the form of Table 1 and the histogram Fig. 1.

Table 1

The probability distribution of the spare parts

\begin{tabular}{|l|l|l|}
\hline \multicolumn{1}{|c|}{$\boldsymbol{y}$} & \multicolumn{1}{c|}{$\boldsymbol{p}(\boldsymbol{y})$} & \multicolumn{1}{c|}{$\boldsymbol{F}(\boldsymbol{y})$} \\
\hline 0 & 0,03 & 0,03 \\
\hline 1 & 0,14 & 0,17 \\
\hline 2 & 0,22 & 0,39 \\
\hline
\end{tabular}




\begin{tabular}{|l|l|l|}
\hline 3 & 0,27 & 0,66 \\
\hline 4 & 0,19 & 0,85 \\
\hline 5 & 0,11 & 0,96 \\
\hline 6 & 0,04 & 1 \\
\hline
\end{tabular}

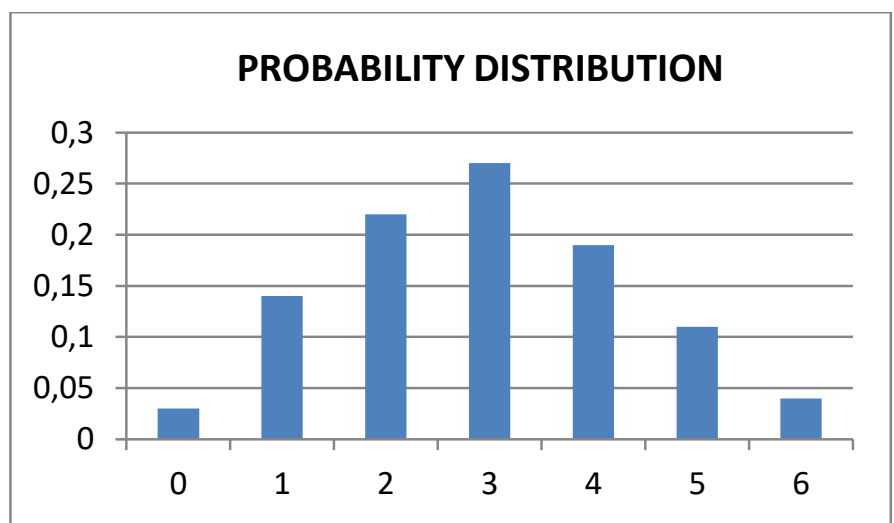

Fig. 1. Histogram of the probability of using spare parts

\section{A pseudo-random number generator}

The main task of this stage is Monte Carlo simulation calculations by means of pseudo - random numbers from the interval $(0.1)$ of the uniform distribution. The basic mathematical operation used in the pseudo - random number generator is the socalled modulo operation (9) (Patrykiejew, 1998; Janke, 2002):

$$
y=x \bmod m
$$

where:

$y$ is the remainder of division $x$ by $m$.

The numbers generated by the generator, developed in VBA for Application based on the cumulative inversion method, are transformed into the form of the desired distribution of the variable numbers of spare parts replacements (L'ecuyer, 1990, 2004). The randomly determined size of y variable number of replacements of spare parts makes it easier to make decisions about creating an appropriate stock of these parts during current production (Fig. 2). 


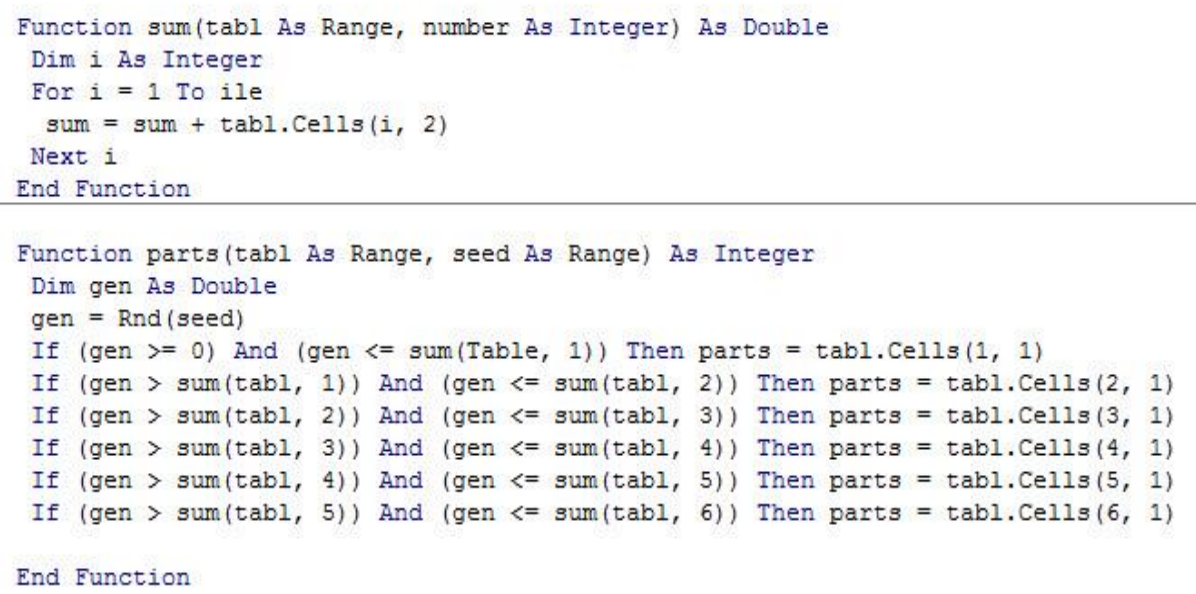

Fig. 2. The pseudorandom number generator code

Source: (Jackson and Staunton, 2004; Walkenbach, 2010; Matloff, 2006)

In order to determine the optimal solution, i.e. to determine the amount of spare parts, which minimizes the expected loss of the producer and the user, additional data were assumed: $c_{s}=430 € ; c_{c}=760 € ; c_{d m}=850 €$. By entering data into the formula (8), it was obtained:

$$
\begin{gathered}
F_{p-1}<\frac{c_{c}-c_{s}+c_{d m}}{c_{s}+c_{d m}}<F_{p} \\
F_{p-1}<0,73<F_{p}
\end{gathered}
$$

The following solution was obtained:

Table 2

Determining the optimal number of spare parts

\begin{tabular}{|l|l|l|}
\hline \multicolumn{1}{|c|}{$\boldsymbol{y}$} & \multicolumn{1}{|c|}{$\boldsymbol{p}(\boldsymbol{y})$} & \multicolumn{1}{c|}{$\boldsymbol{F}(\boldsymbol{y})$} \\
\hline 0 & 0,03 & 0,03 \\
\hline 1 & 0,14 & 0,17 \\
\hline 2 & 0,22 & 0,39 \\
\hline 3 & 0,27 & 0,66 \\
\hline 4 & 0,19 & 0,85 \\
\hline 5 & 0,11 & 0,96 \\
\hline 6 & 0,04 & 1 \\
\hline
\end{tabular}

\section{CONCLUSIONS}

The developed method for controlling the stock of spare parts made it possible to determine the economic size of stocks during the production of current short-run devices on a given example. For the adopted statistical data, the result of 4 parts for 
the device was obtained (tab. 2). The quantity of spare parts determined in this way can be verified by a pseudo-random number generator, which randomly analyzes various damage scenarios and, consequently, the number of parts replacements, selects the optimal quantities. The research carried out confirms two tendencies to improve the efficiency of production processes: by depleting production or by making the production process more flexible and adaptable to dynamically changing market needs. It should also be noted that one of the fundamental factors affecting the efficiency of the production process is maintaining the continuity of material flow. For this reason, the issue of spare parts used to maintain traffic is becoming more and more important (Koliński, 2011; Kolińska, 2013).

\section{REFERENCES}

APICS Dictionary, 2004. 11th Edition, American Production and Inventory Control Society, Inc., Falls Church, VA.

DIN 24420, 1976. Deutsches Institut Für Normung.

Grondys, K., 2014. Stocks management of spare parts based on the concept of a common warehouse. Przegląd Organizacji, 5.

Jackson, M., Staunton, M., 2004. Advanced financial models using Excel and VBA, Helion, Gliwice.

Janke, W., 2002. Pseudo Random Numbers: Generation and Quality Checks, Quantum Simulations of Complex Many-Body Systems: From Theory to Algorithms. In: Lecture Notes, J. Grotendorst, D. Marx, A. Muramatsu (Eds.), John von Neumann Institute for Computing, Julich, NIC Series, Vol. 10, ISBN 3-00009057-6.

Kolińska, K., 2013. Efficiency of inventory management process of spare parts in manufacturing enterprises - research results. Gospodarka Materiałowa i Logistyka, 3.

Koliński, A., 2011. Review of methods and techniques for assessing the effectiveness of the production process. Logistyka, 5.

L'Ecuyer, P., 2004. Working Paper Random number generation. Papers / HumboldtUniversität Berlin, Center for Applied Statistics and Economics (CASE), No 21.

L'ecuyer, P., 1990. Random numbers for simulation. Communications of the ACM, 33, $10,85-97$.

Matloff, N., 2006. Random Number Generation, N.S. Matloff.

Patrykiejew, A., 1998. Introduction to the Monte Carlo method, UMCS, Lublin.

Sarjusz-Wolski, Zd., 2000. Supply Management Strategy, PWE, Warszawa.

Ślaski, P., 2017. Inventory Management in Logistic Systems, WAT, Warszawa.

Walkenbach, J., 2010. Power Programming Exce/® 2010 with VBA, Wiley Publishing, Indianapolis, Indiana.

Krzaczak, P., Stoma, M., Dudziak, A., 2012. Problems of logistics of spare parts in the aspect of maintaining agricultural tractors in a technical readiness. Logistyka, 4, Poznań. 